\title{
Pulmonary artery pressure is associated with mid-term major adverse cardiovascular events and postprocedure pericardial effusion in atrial fibrillation patients undergoing left atrial appendage occlusion
}

\author{
Tian Zou ${ }^{1 \#}$, Qingxing Chen ${ }^{1 \#}$, Lei Zhang ${ }^{1 *}$, Chaofeng Chen ${ }^{1}$, Yunlong Ling ${ }^{1}$, Guijian Liu ${ }^{1}$, \\ Sunying Wang ${ }^{2}$, Yang Pang ${ }^{1}$, Ye Xu ${ }^{1}$, Kuan Cheng ${ }^{1}$, Daxin Zhou ${ }^{1}$, Wenqing Zhu ${ }^{1}$, Junbo Ge ${ }^{1}$ \\ ${ }^{1}$ Department of Cardiology, Zhongshan Hospital, Fudan University, Shanghai Institute of Cardiovascular Disease, Shanghai, China; ${ }^{2}$ Department of \\ Cardiology, Shengli Clinical Medical College of Fujian Medical University, Fuzhou, China \\ Contributions: (I) Conception and design: T Zou, Q Chen, L Zhang; (II) Administrative support: D Zhou, W Zhu, J Ge; (III) Provision of study \\ materials or patients: T Zou, Q Chen, L Zhang, C Chen, Y Ling, G Liu; (IV) Collection and assembly of data: T Zou, Q Chen, L Zhang, S Wang, \\ Y Pang, Y Xu, K Cheng; (V) Data analysis and interpretation: T Zou, Q Chen, L Zhang, C Chen, Y Ling, G Liu, D Zhou, W Zhu; (VI) Manuscript \\ writing: All authors; (VII) Final approval of manuscript: All authors.
}

\#These authors contributed equally to this work.

Correspondence to: Prof. Wenqing Zhu, MD, PhD; Prof. Daxin Zhou, MD, PhD. Department of Cardiology, Zhongshan Hospital, Fudan University, Shanghai 200032, China. Email: zhu.wenqing@zs-hospital.sh.cn; zhou.daxin@zs-hospital.sh.cn.

Background: Patients with nonvalvular atrial fibrillation (NVAF) undergoing left atrial appendage occlusion (LAAO) are at high risk of stroke or bleeding. However, risk factors for their adverse cardiovascular events remain largely unknown. Pulmonary hypertension has been shown to be related to poor prognosis in many heart diseases. In this study, we determined whether elevated pulmonary artery systolic pressure (PASP) is associated with postprocedure adverse events and major adverse cardiovascular events (MACE) in these patients.

Methods: From June 2017 and December 2019, 530 consecutive patients with NAVF at high risk of stroke or bleeding who undergone LAAO were retrospectively enrolled in our study. The preprocecure PASP was obtained by transthoracic echocardiography using the simplified Bernoulli's equation. Patients were followed-up through clinic visits or over the phone at discharge at 1-3 months, 6 months, and annually thereafter. The median follow-up time was 12 months, and clinical data were analyzed. MACE was defined as myocardial infarction, definite heart failure, stroke, or all-cause death. The outcome of postprocedure pericardial effusion included in-hospital pericardial effusion and pericardial effusion detected after discharge. Results: Univariate analyses indicated that patients who had MACE tended to have elevated PASP $(\mathrm{P}=0.005)$. After dividing the cohort according to the cut-off value of PASP, Kaplan-Meier curves indicated that patients with PASP $\geq 39.5 \mathrm{mmHg}$ had a higher risk of MACE $(\mathrm{P}=0.007)$ and heart failure hospitalization $(\mathrm{P}=0.005)$ compared to patients whose PASP $<39.5 \mathrm{mmHg}$. Cox regression analysis showed that PASP was a predominant risk factor of MACE ( $\mathrm{HR}=2.337,95 \% \mathrm{CI}, 1.207-4.526, \mathrm{P}=0.012)$ and heart failure hospitalization ( $\mathrm{HR}=3.701,95 \% \mathrm{CI}, 1.118-12.251, \mathrm{P}=0.032$ ). Furthermore, the PASP cut-off added incremental discriminatory capacity to the MACE risk model of this cohort. In addition, logistic regression showed that PASP had as a significant association with postprocedure pericardial effusion $(\mathrm{OR}=1.061$, $\mathrm{P}=0.032$ ).

Conclusions: Elevated PASP was associated with postprocedure pericardial effusion and mid-term MACEs in patients with atrial fibrillation (AF) undergoing LAAO.

Keywords: Atrial fibrillation (AF); pulmonary artery systolic pressure (PASP); prognosis; left atrial appendage occlusion (LAAO) 
Submitted Jun 17, 2021. Accepted for publication Aug 01, 2021.

doi: $10.21037 /$ atm-21-3561

View this article at: https://dx.doi.org/10.21037/atm-21-3561

\section{Introduction}

Atrial fibrillation (AF) is the most common arrhythmia worldwide, with a significant impact on both morbidity and mortality (1). AF patients at high risk of stroke or bleeding are often recommended to receive left atrial appendage occlusion (LAAO) (2). This cohort of AF patients undergoing LAAO share risk factors of major adverse cardiovascular events (MACE), major risk factors of which including age, obesity, history of stroke, history of atrial fibrillation, New York Heart Association (NYHA) class, $\mathrm{N}$-terminal pro brain natriuretic peptide (NT-proBNP) and pulmonary artery systolic pressure (PASP) (3-6).

Among them, PASP measured by transthoracic echocardiography is a non-invasive method routinely quantified in clinical practice (7-9). As a known complication of left-sided heart disease, pulmonary hypertension is related to poor outcomes, even with mild elevation in pulmonary pressure (10-12). Previous studies have identified the association between pulmonary hypertension and multiple adverse events of the heart failure and hypertrophic cardiomyopathy cohorts $(3,13,14)$. From the perspective of pathophysiology, frequently onset of AF leads to sustained elevation of left atrial pressure. It may cause alveolar-capillary network destruction and pulmonary vascular remodeling, consequently leading to pulmonary arterial hypertension and right ventricular dysfunction or failure. Following LAAO, dilated pulmonary artery induced by pulmonary arterial hypertension may increase the friction between the pulmonary artery and the occlusion device, which may account for subsequent perforation or reactive pericardial effusion.

In this study, we aimed to determine whether elevated PASP is associated with postprocedure adverse events and MACE in AF patients undergoing LAAO, thus elucidate the implications of PASP on the prognosis of high risk cohort of AF patients. We present the following article in accordance with the STROBE reporting checklist (available at https://dx.doi.org/10.21037/atm-21-3561).

\section{Methods}

\section{Study population}

Using a retrospective cohort study design, we identified consecutive patients with $\mathrm{AF}$ who successfully underwent LAAO using the Watchman device at Zhongshan Hospital, Fudan University between June 2017 and December 2019. The inclusion criteria included those patients aged 18 years and older, whose CHA2DSVASC $\geq 2$ or HAS-BLED $\geq 3$. The exclusion criteria included patients who were diagnosed with valvular AF. Finally, 530 patients were included in our study cohort.

All procedures performed in this study involving human participants were in accordance with the Declaration of Helsinki (as revised in 2013). The study was approved by the Institutional Review Board of Zhongshan Hospital, Fudan University, Shanghai, China (B2021-042) and individual consent for this retrospective analysis was waived.

\section{Echocardiography}

All patients received a transthoracic echocardiography 1-7 days prior to LAAO. The PASP was obtained by echocardiography using the simplified Bernoulli's equation: $\left[\mathrm{PASP}=4 \times(\text { peak tricuspid regurgitant velocity })^{2}+\right.$ mean right atrial pressure]. Measurements were made by experienced cardiac sonographers who were blinded to the clinical data.

\section{Procedure and follow-up}

Other clinical data, including age, gender, BMI, medical history, laboratory measures, echocardiographic parameters and postprocedure medication were analyzed, and no missing data was detected across all variables.

All patients in our cohort were implanted with the Watchman device in accordance with descriptions detailed in previous studies (15). Intra-procedural imaging assessed the left atrial appendage size and excluded the presence of intracardiac thrombi. A routine 60-day course of oral anticoagulants was recommended, with postprocedure follow-ups at 1-3 months, 6 months, and annually thereafter. Patients were followed-up through clinic visits or over the phone at discharge.

In our study, MACE was defined as myocardial infarction, definite heart failure, stroke, or all-cause death.

The outcome of postprocedure pericardial effusion 
included in-hospital pericardial effusion and pericardial effusion detected after discharge.

\section{Statistical analysis}

Continuous values were reported as mean \pm standard deviation (SD) when normally distributed, and as median and interquartile range when skewed. Categorical variables were reported as frequencies and percentages. An independent-sample $t$-test was used to identify the differences in continuous variables [age, body mass index (BMI), eGFR, left atrial diameter (LAD), hemoglobin] and Kruskal-Wallis test was used to identify the differences in continuous variables [Troponin I, NT-proBNP, Creatinine, high sensitive C-reactive protein (hsCRP), D-dimer, left ventricular ejection fraction (LVEF), PASP]. Chi-square test was used to identify differences in categorical variables. The PASP value was investigated using a receiver operating characteristics (ROC) curve to determine the optimal cutoff value for the prediction of MACE. The optimal cutoff was defined as the point of the highest Youden index (Youden index $=$ sensitivity + specificity -1 ). The event free survival rate was analyzed using a Kaplan-Meier survival curve, and log-rank tests were applied for comparison. The covariates used for adjustment were as follows: age and gender in model 1; age, gender, BMI, history of diabetes, history of hypertension, history of paroxysmal AF, history of ischaemic vascular disease, history of coronary heart disease, history of stroke, history of chronic heart failure in model 2; age, gender, BMI, history of diabetes, history of hypertension, history of paroxysmal AF, history of ischaemic vascular disease, history of coronary heart disease, history of stroke, history of chronic heart failure, NYHA class, LVEF, LAD, troponin I, NT-proBNP, Creatinine, hsCRP, D-dimer, and Hemoglobin in model 3; and age, body mass index, history of stroke, NYHA class, NT-proBNP, eGFR, hsCRP and PASP in model 4. Models 1, 2, 3 and 4 were used to estimate the risk of MACE and heart failure hospitalization separately. Multivariate Cox proportional hazards analysis and area under the ROC curve (AUC) were applied to evaluate the discriminatory capacity of the models for predicting the risk of MACE and heart failure hospitalization. Logistic regression and univariate analyses were applied to predict postprocedure pericardial effusion. No missing data was detected across all variables. All statistical analyses were performed using SPSS software, version 24 software (SPSS; Chicago, IL, USA). The twosided $\mathrm{P}<0.05$ was consider statistically significant.

\section{Results}

\section{Patient characteristics and follow-up results}

After excluding patients who reached the exclusion criteria and those lost to follow-up, 530 patients were included in the final study cohort. Data on all baseline variables were analyzed. The average age of this cohort was $69 \pm 9$ years. Overall, $62.9 \%$ patients were male, $282(41.8 \%)$ had a history of paroxysmal AF, 233 (43.9\%) had a history of stroke, and $110(20.7 \%)$ had a history of chronic heart failure. The median follow-up time was 12 months (range, 6-47 months). During the follow-up period, a total of 39 patients reached the MACE end points, 14 patients were readmitted to hospital for heart failure, three patients died of heart failure, and one patient died of stroke. Generally, patients who had MACE tended to be older $(\mathrm{P}=0.017)$, and had elevated NT-proBNP ( $\mathrm{P}=0.023)$, hsCRP $(\mathrm{P}=0.018)$, and PASP $(\mathrm{P}=0.005)$ (Table 1).

\section{PASP and mid-term adverse events}

ROC curve analysis showed that the optimal PASP cutoff value for the prediction of MACE occurrence was $39.5 \mathrm{mmHg}$, with a specificity of $68.9 \%$ and a sensitivity of $56.4 \%$. Based on this cut-off value, the patients were divided into two groups: there were $175(33.0 \%)$ patients in the PASP $\geq 39.5 \mathrm{mmHg}$ group and 355 (67.0\%) patients in the PASP $<39.5 \mathrm{mmHg}$ group. The Kaplan-Meier curves indicated that patients with PASP $\geq 39.5 \mathrm{mmHg}$ had a higher risk of MACE $(\mathrm{P}=0.007)$ and heart failure hospitalization $(\mathrm{P}=0.005)$ compared to those with a PASP $<39.5 \mathrm{mmHg}$ (Figure 1). Variables identified through univariable screening, along with some conventional risk factors, were entered into three multivariable models. Multivariate Cox proportional hazards analysis showed that PASP was a predominant risk factor of MACE (hazard ratio $=2.337,95 \%$ confidence interval, $1.207-4.526, \mathrm{P}=0.012$ ) and heart failure hospitalization (hazard ratio $=3.701,95 \%$ confidence interval, 1.118-12.251, $\mathrm{P}=0.032$ ) (Table 2).

\section{The predictive value of models includes PASP}

A risk stratification model was constructed to predict MACE and heart failure readmission. Figure $2 A$ and Table 3 showed that adding the PASP cut-off to model 4 was slightly more predictive than model 4 alone in predicting MACE, with an AUC of 0.713 , which is an increase of 0.043 $(\mathrm{P}=0.025)$. Figure $2 B$ and Table 3 showed that adding the 
Table 1 Baseline characteristics between patients with and without MACE

\begin{tabular}{|c|c|c|c|}
\hline Characteristics & No MACE $(n=491)$ & MACE $(n=39)$ & $P$ value \\
\hline \multicolumn{4}{|l|}{ Demographics } \\
\hline Age (years) & $68.5 \pm 8.8$ & $72.0 \pm 8.9$ & 0.017 \\
\hline Gender (male), n (\%) & $311(63.3)$ & $23(60.0)$ & 0.587 \\
\hline BMI $\left(\mathrm{kg} / \mathrm{m}^{2}\right)$ & $24.9 \pm 3.3$ & $24.4 \pm 3.3$ & 0.439 \\
\hline Hypertension & $327(66.6)$ & $28(71.8)$ & 0.507 \\
\hline Diabetes & $99(25.3)$ & $8(20.5)$ & 0.958 \\
\hline Paroxysmal atrial fibrillation & $206(42.0)$ & $21(53.8)$ & 0.910 \\
\hline Ischaemic vascular disease & $41(8.4)$ & $7(18.0)$ & 0.086 \\
\hline NYHA class 2 & $90(18.3)$ & $7(17.9)$ & 0.097 \\
\hline NYHA class 3 & $13(2.6)$ & $4(10.3)$ & \\
\hline NYHA class 4 & $1(0.2)$ & $0(0.0)$ & \\
\hline \multicolumn{4}{|l|}{ Laboratory measures } \\
\hline Troponin I (ng/mL) & $0.011(0.008-0.018)$ & $0.012(0.009-0.018)$ & 0.318 \\
\hline NT-proBNP (pg/mL) & $676.5(340.0-1,290.0)$ & $871.0(559.0-1,411.0)$ & 0.023 \\
\hline Creatinine $(\mu \mathrm{mmol} / \mathrm{L})$ & $81.0(69.0-94.0)$ & $84.0(61.0-221.3)$ & 0.789 \\
\hline LVEF (\%) & $64[60-67]$ & $66[60-67]$ & 0.153 \\
\hline LAD (mm) & $48.5 \pm 8.4$ & $49.7 \pm 6.5$ & 0.427 \\
\hline PASP $(\mathrm{mmHg})$ & $35[31-41]$ & $41[35-43]$ & 0.005 \\
\hline \multicolumn{4}{|l|}{ Postprocedure medication, n (\%) } \\
\hline Warfarin & $35(7.1)$ & $2(5.1)$ & 0.884 \\
\hline Rivaroxaban & $287(58.5)$ & $18(46.2)$ & 0.135 \\
\hline Dabigatran & $81(16.9)$ & $9(23.1)$ & 0.292 \\
\hline Aspirin & $298(60.7)$ & $22(56.4)$ & 0.599 \\
\hline Class I antiarrhythmic drugs & $25(5.1)$ & $3(7.7)$ & 0.744 \\
\hline Class III antiarrhythmic drugs & $108(22.0)$ & $7(17.9)$ & 0.555 \\
\hline Beta-blockers & $202(41.1)$ & 19 (58.9) & 0.356 \\
\hline
\end{tabular}

BMI, body mass index; NYHA, New York Heart Association; NT-pro BNP, N-terminal pro brain natriuretic peptide; eGFR, estimate glomerular filtration rate; hsCRP, high sensitive C-reactive protein; LAD, left atrial diameter; LVEF, left ventricular ejection fraction; PASP, pulmonary artery systolic pressure. 

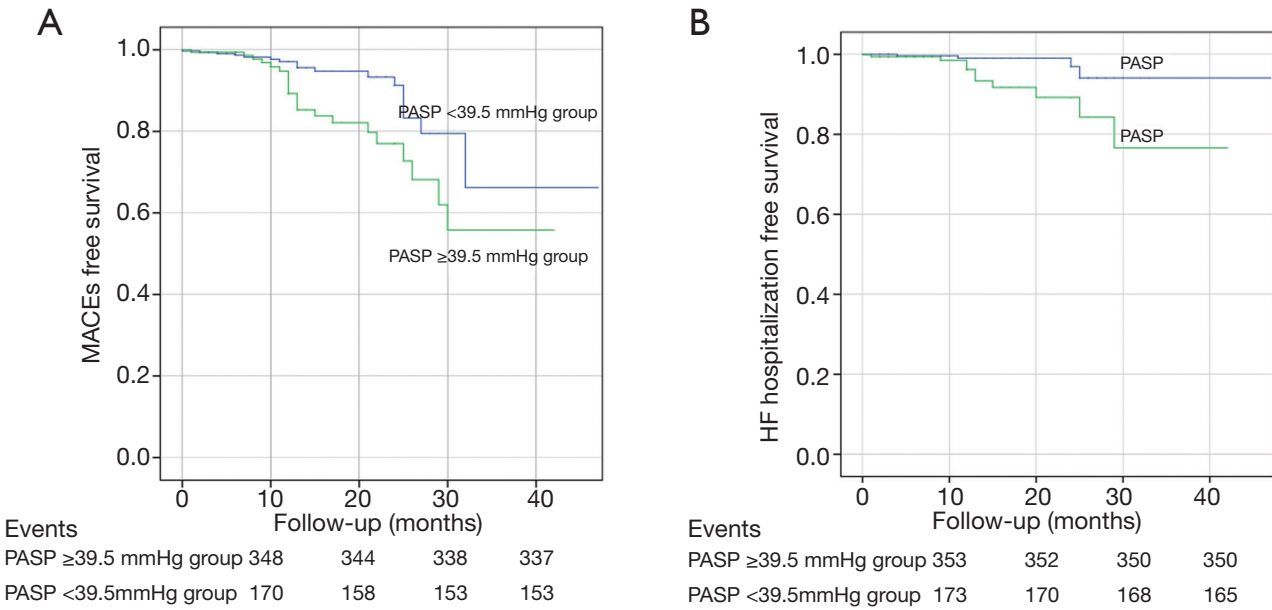

Figure 1 Kaplan-Meier estimates of mid-term clinical outcomes according to the PASP cut-off. PASP, pulmonary artery systolic pressure.

Table 2 Risk of mid-term events for PASP $\geq 39.5 \mathrm{mmHg}$

\begin{tabular}{lcc}
\hline Variables & $\begin{array}{c}\text { Adjusted hazard ratio } \\
\text { (95\% confidence interval) }\end{array}$ & P value \\
\hline MACE & $2.333(0.127-4.400)$ & 0.009 \\
Model 1 & $2.792(1.456-5.357)$ & 0.002 \\
Model 2 & $2.337(1.207-4.526)$ & 0.012 \\
Model 3 & $4.583(0.1436-14.631)$ & 0.010 \\
Heart failure readmission & 0.030 \\
Model 1 & $6.251(1.884-20.737)$ & 0.032 \\
Model 2 & $3.701(1.118-12.251)$ & \\
\hline
\end{tabular}

Model 1: adjusted for age and gender. Model 2: adjusted for age, gender, body mass index, history of diabetes, history of hypertension, history of paroxysmal atrial fibrillation, history of ischaemic vascular disease, history of coronary heart disease, history of stroke, and history of chronic heart failure. Model 3: adjusted for age, gender, body mass index, history of diabetes, history of hypertension, history of paroxysmal atrial fibrillation, history of ischaemic vascular disease, history of coronary heart disease, history of stroke, history of chronic heart failure, NYHA class, LVEF, LAD, troponin I, NT-proBNP, creatinine, hsCRP, D-dimer, and hemoglobin. PASP, pulmonary artery systolic pressure; NYHA, New York Heart Association; LVEF, left ventricular ejection fraction; LAD, left atrial diameter; hsCRP, high sensitive C-reactive protein.
PASP cut-off to model 4 was also slightly more predictive than model 4 alone in predicting heart failure readmission, with an AUC of 0.784, which is an increase of 0.0450 $(\mathrm{P}=0.090)$.

\section{PASP and postprocedure pericardial effusion}

Fourteen $(2.6 \%)$ patients had postprocedure pericardial effusion. Among them, 11 patients had postprocedure pericardial effusion during postprocedure hospitalization, while the other three patients had postprocedure pericardial effusion within 1 year after the procedure. Five patients received a conservative treatment, six patients underwent a pericardiocentesis, and three patients underwent surgical treatment. Fortunately, all of these patients recovered after their treatment. To investigate the associations between PASP and postprocedure pericardial effusion, univariate and logistic regression analyses were applied to the echocardiographic parameters and procedure characteristics. As shown in Table 4, PASP had a significant association with postprocedure pericardial effusion after multivariate adjustment [unadjusted hazard ratio $=1.057$, 95\% confidence interval (1.002-1.115), $\mathrm{P}=0.041$; adjusted hazard ratio $=1.061,95 \%$ confidence interval $(1.005-1.120)$, $\mathrm{P}=0.032]$. 

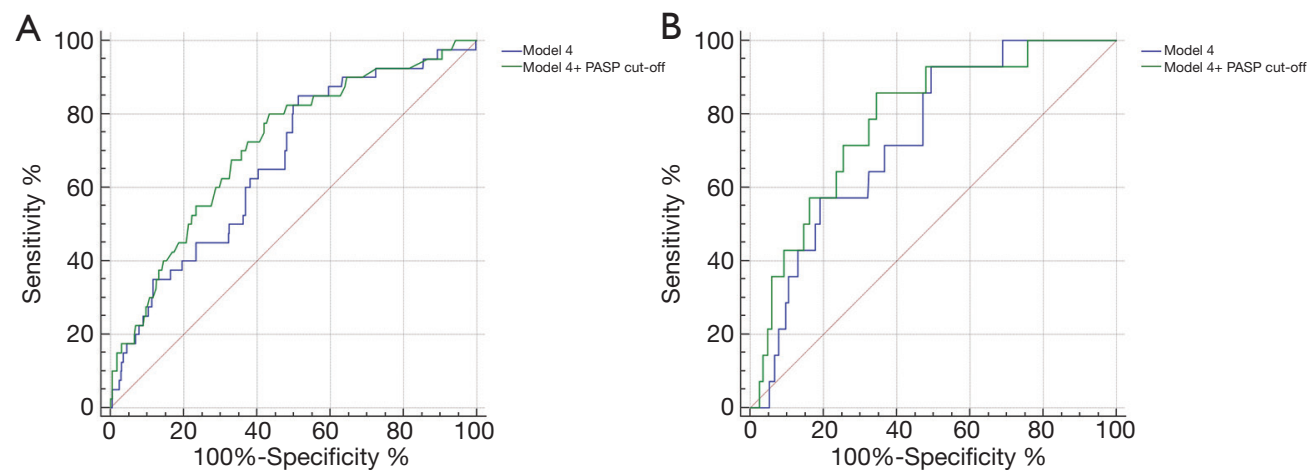

Figure 2 Area under the receiver operating characteristic curve of models predicting clinical outcomes. (A) Area under the receiver operating characteristic curve of models predicting MACE. (B) Area under the receiver operating characteristic curve of models predicting heart failure readmission. MACE, major adverse cardiovascular event.

Table 3 Area under the receiver operating characteristic curves of models predicting MACE and heart failure readmission

\begin{tabular}{|c|c|c|c|}
\hline Variables & Model 4 & Mode 4 + PASP cut-off & $P$ value \\
\hline Heart failure readmission, AUC (95\% confidence interval) & $0.734(0.694-0.772)$ & $0.784(0.746-0.818)$ & 0.090 \\
\hline
\end{tabular}

Model 4: adjusted for age, body mass index, history of stroke, NYHA class, NT-proBNP, eGFR, hsCRP and PASP.

Table 4 Univariate and multivariate analyses of the association with postprocedure pericardial effusion

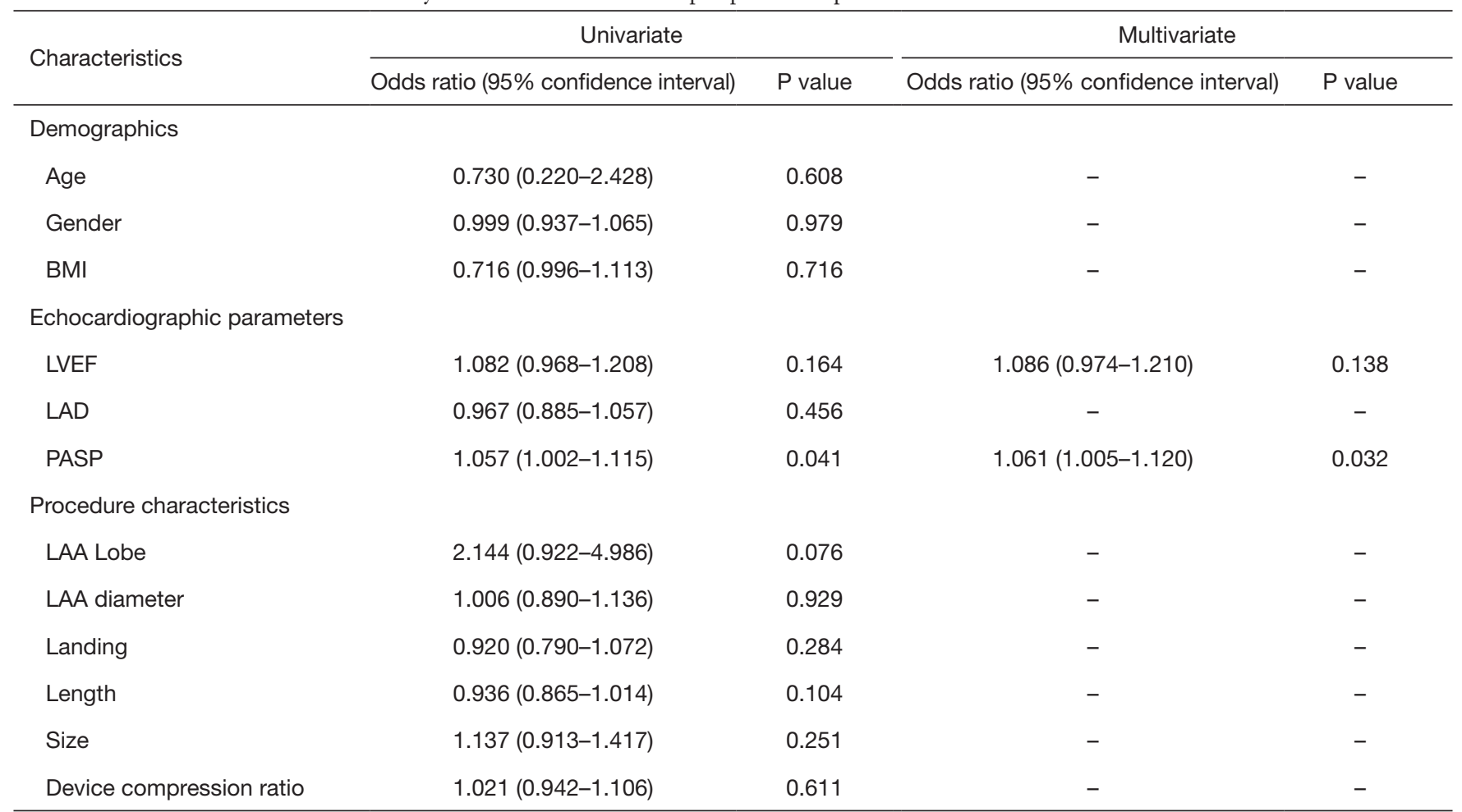

LAD, left atrial diameter; LVEF, left ventricular ejection fraction; PASP, pulmonary artery systolic pressure; LAA, Left atrial appendage. 


\section{Discussion}

The main findings of our current study were as follows: (I) for $\mathrm{AF}$ patients at high risk of stroke or bleeding who underwent LAAO, PASP $\geq 39.5 \mathrm{mmHg}$ was an independent risk factor of composite outcomes of MACE or heart failure hospitalization during the mid-term follow-up period; (II) high PASP was a risk factor of postprocedure pericardial effusion in AF patients who underwent LAAO. Our study provides important information on the cardiovascular prognosis value of PASP in the high-risk AF cohort. Furthermore, to the best of our knowledge, our study was the first to demonstrate an association between pulmonary artery pressure and postprocedure pericardial effusion in LAAO.

Although invasive right heart catheterization is the gold standard for assessment of pulmonary artery pressure, PASP measured by echocardiography has shown a linear correlation compared to PASP detected via an invasive method (16-20). Therefore, PASP, which is routinely quantified in clinical echocardiography, is also validated as a useful parameter in clinical practice. In previous studies, the definition thresholds of PASP in the diagnosis of pulmonary artery hypertension vary $(7,8,21,22)$; however, the PASP cutoff value of $39.5 \mathrm{mmHg}$ in our study is close to $40 \mathrm{mmHg}$, which is the acknowledged cut-off in some classic studies $(14,23)$. Overall, elevated PASP (PASP $\geq 39.5 \mathrm{mmHg}$ ) was present in a significant proportion $(33.0 \%)$ of our highrisk $\mathrm{AF}$ cohort. This trend is approximate to the prevalence reported in the AF with heart failure with preserved left ventricular ejection fraction (HFpEF) cohort (24), but is higher than that of the AF cohort without a prior heart failure hospitalization (4).

Elevated pulmonary artery pressure, as a risk factor of heart failure hospitalization, all-cause mortality, and cardiovascular mortality, has been reported in many heart diseases. In the hypertrophic cardiomyopathy cohort, PASP was associated with increased stroke, systemic embolism, and all-cause mortality in both obstructive and nonobstructive circumstances $(13,14)$. Elevated preoperative PASP was associated with worse postoperative LVEF and increased mortality in patients undergoing mitral valve surgery for severe mitral regurgitation $(6,25)$. High PASP has also been consistently and independently prognostic of heart failure-related events in the HFpEF cohort $(3,26)$. Interestingly, $\mathrm{AF}$ and $\mathrm{HFpEF}$ are highly prevalent diseases with similar risk factors (27), which might imply that the prediction of cardiovascular events by PASP could also be reasonable.
Our findings suggest that PASP was an independent prognostic factor of heart failure hospitalization and MACE in high-risk AF patients undergoing LAAO, further improving the cardiovascular risk models developed in our study. The pathophysiological connection between PASP and cardiovascular events might consist of the following factors: AF leads to haemodynamic perturbations in the left atrium, cumulatively resulting in the elevation of left atrial pressure (28-30). Sustained elevation of left atrial pressure may cause alveolar-capillary network destruction and pulmonary vascular remodeling, consequently leading to irreversible pulmonary arterial hypertension and right ventricular dysfunction or failure $(31,32)$. Furthermore, pulmonary arterial hypertension represents the downstream effect of AF, and as such, was seen with increased onset frequency and associated with a more ominous prognosis in this setting.

Previous studies reported that age, obesity, and high stroke risk were related to pericardial effusion requiring intervention of LAAO $(33,34)$. Nevertheless, the underlying correlation between echocardiographic parameters and pericardial effusion remains elusive. Several case reports (35-37) have documented incidents of pericardial tamponade due to device erosion into the pulmonary artery by tip of the strut or bards of device. However, it is unclear whether pulmonary artery anatomical structures are related to this complication.

Our findings indicate that PASP was a risk factor of postprocedure (including in-hospital and after discharge) pericardial effusion in AF patients with LAAO. Halkin et al. (38) analyzed cardiac-gated computed tomography (CCTA) of $100 \mathrm{AF}$ patients, and found only 7\% complete separation between the pulmonary artery and the left atrial appendage. Therefore, in most cases, left atrial appendage is anatomically adjacent to the pulmonary artery, and is in direct contact with the main pulmonary artery. A dilated pulmonary artery induced by pulmonary arterial hypertension may further increase the friction between the pulmonary artery and the occlusion device, which may account for subsequent perforation or reactive pericardial effusion.

This finding suggests an awareness of especially flexible operation and a relatively lower device compression ratio in patients with elevated PASP undergoing LAAO (39). Compared with the Amplatzer Cardiac Plug, the Watchman device with shorter barbs and smaller penetration depth is considered to be safer for patients with elevated PASP. In addition, CCTA may be important for preoperative 
evaluation of left atrial appendage and its adjacent anatomical structure.

\section{Limitations}

Our study has several limitations that should be noted. Firstly, this was a single-centre retrospective observational study, which may have resulted in certain measurement bias in the data collection and limitation in sample representativeness. Secondly, despite being confirmed via a linear correlation with a gold standard, PASP was still an estimated value derived from echocardiography, as opposed to a more accurate invasive right heart catheterization method. Thirdly, the number of patients who reached the endpoint in this study was relatively small. Therefore, risk factors of cardiac death and all-cause mortality could not be analyzed in this study. Furthermore, when assessing the discriminatory ability of the regression model, we have to reduce the risk factors in model 3 in case of over-fitting, and come up with model 4 according to overall consideration of our data analysis and relevant articles (3-6). It is possible that the risk factors may have been underestimated in multivariate analysis.

\section{Conclusions}

For AF patients at high risk of stroke or bleeding who underwent LAAO, elevated PASP was an independent risk factor of the composite outcomes of MACE or heart failure hospitalization during mid-term follow-up. Furthermore, high PASP was also a risk factor of postprocedure pericardial effusion in AF patients who underwent LAAO.

\section{Acknowledgments}

Tian Zou especially wishes to thank her families and Jun Yan, her boyfriend, for giving her spiritual support over the past years.

Funding: This work was supported by the Project of Shanghai Science and Technology Committee (Nos.: 17DZ1930102, 17DZ1930303) and the scientific research funding of Zhongshan Hospital, Fudan University (No.: 331).

\section{Footnote}

Reporting Checklist: The authors have completed the STROBE reporting checklist. Available at https://dx.doi. org/10.21037/atm-21-3561
Data Sharing Statement: Available at https://dx.doi. org/10.21037/atm-21-3561

Conflicts of Interest: All authors have completed the ICMJE uniform disclosure form (available at https://dx.doi. org/10.21037/atm-21-3561). The authors have no conflicts of interest to declare.

Ethical Statement: The authors are accountable for all aspects of the work in ensuring that questions related to the accuracy or integrity of any part of the work are appropriately investigated and resolved. All procedures performed in this study involving human participants were in accordance with the Declaration of Helsinki (as revised in 2013). The study was approved by the Institutional Review Board of Zhongshan Hospital, Fudan University, Shanghai, China (B2020-042) and individual consent for this retrospective analysis was waived.

Open Access Statement: This is an Open Access article distributed in accordance with the Creative Commons Attribution-NonCommercial-NoDerivs 4.0 International License (CC BY-NC-ND 4.0), which permits the noncommercial replication and distribution of the article with the strict proviso that no changes or edits are made and the original work is properly cited (including links to both the formal publication through the relevant DOI and the license). See: https://creativecommons.org/licenses/by-nc-nd/4.0/.

\section{References}

1. DeLago AJ, Essa M, Ghajar A, et al. Incidence and Mortality Trends of Atrial Fibrillation/Atrial Flutter in the United States 1990 to 2017. Am J Cardiol 2021;148:78-83.

2. January CT, Wann LS, Calkins H, et al. 2019 AHA/ ACC/HRS Focused Update of the 2014 AHA/ACC/ HRS Guideline for the Management of Patients With Atrial Fibrillation: A Report of the American College of Cardiology/American Heart Association Task Force on Clinical Practice Guidelines and the Heart Rhythm Society in Collaboration With the Society of Thoracic Surgeons. Circulation 2019;140:e125-51.

3. Suzuki S, Kaikita K, Yamamoto E, et al. H2FPEF score for predicting future heart failure in stable outpatients with cardiovascular risk factors. ESC Heart Failure 2020;7:66-75.

4. Prapan N, Ratanasit N, Karaketklang K. Significant functional tricuspid regurgitation portends poor outcomes 
in patients with atrial fibrillation and preserved left ventricular ejection fraction. BMC Cardiovasc Disord 2020;20:433.

5. Cicek V, Cinar T, Hayiroglu MI, et al. Preoperative cardiac risk factors associated with in-hospital mortality in elderly patients without heart failure undergoing hip fracture surgery: a single-centre study. Postgrad Med J 2020. doi: 10.1136/postgradmedj-2020-138679. [Epub ahead of print].

6. Varghese R, Itagaki S, Anyanwu AC, et al. Predicting early left ventricular dysfunction after mitral valve reconstruction: the effect of atrial fibrillation and pulmonary hypertension. J Thorac Cardiovasc Surg 2014;148:422-7.

7. Galiè N, Humbert M, Vachiery JL, et al. 2015 ESC/ERS Guidelines for the diagnosis and treatment of pulmonary hypertension: The Joint Task Force for the Diagnosis and Treatment of Pulmonary Hypertension of the European Society of Cardiology (ESC) and the European Respiratory Society (ERS): Endorsed by: Association for European Paediatric and Congenital Cardiology (AEPC), International Society for Heart and Lung Transplantation (ISHLT). Eur Respir J 2015;46:903-75.

8. Damy T, Goode KM, Kallvikbacka-Bennett A, et al. Determinants and prognostic value of pulmonary arterial pressure in patients with chronic heart failure. Eur Heart J 2010;31:2280-90.

9. Yock PG, Popp RL. Noninvasive estimation of right ventricular systolic pressure by Doppler ultrasound in patients with tricuspid regurgitation. Circulation 1984;70:657-62.

10. Barbieri A, Bursi F, Grigioni F, et al. Prognostic and therapeutic implications of pulmonary hypertension complicating degenerative mitral regurgitation due to flail leaflet: a multicenter long-term international study. Eur Heart J 2011;32:751-9.

11. Bursi F, McNallan SM, Redfield MM, et al. Pulmonary pressures and death in heart failure: a community study. J Am Coll Cardiol 2012;59:222-31.

12. Mandras SA, Mehta HS, Vaidya A. Pulmonary Hypertension: A Brief Guide for Clinicians. Mayo Clin Proc 2020;95:1978-88.

13. Ong KC, Geske JB, Hebl VB, et al. Pulmonary hypertension is associated with worse survival in hypertrophic cardiomyopathy. Eur Heart J Cardiovasc Imaging 2016;17:604-10.

14. Kanbayashi K, Minami Y, Haruki S, et al. Association of elevated pulmonary artery systolic pressure with stroke and systemic embolic events in patients with hypertrophic cardiomyopathy. Int J Cardiol 2017;240:320-3.

15. Zhang X, Jin Q, Kong D, et al. Comparison of fluoroscopy and transesophageal echocardiogram for intra-procedure device surveillance assessment during implantation of Watchman. Int J Cardiol 2021;324:72-7.

16. Berger M, Haimowitz A, Van Tosh A, et al. Quantitative assessment of pulmonary hypertension in patients with tricuspid regurgitation using continuous wave Doppler ultrasound. J Am Coll Cardiol 1985;6:359-65.

17. Bech-Hanssen O, Selimovic N, Rundqvist B, et al. Doppler echocardiography can provide a comprehensive assessment of right ventricular afterload. J Am Soc Echocardiogr 2009;22:1360-7.

18. Currie PJ, Seward JB, Chan KL, et al. Continuous wave Doppler determination of right ventricular pressure: a simultaneous Doppler-catheterization study in 127 patients. J Am Coll Cardiol 1985;6:750-6.

19. Lanzarini L, Fontana A, Lucca E, et al. Noninvasive estimation of both systolic and diastolic pulmonary artery pressure from Doppler analysis of tricuspid regurgitant velocity spectrum in patients with chronic heart failure. Am Heart J 2002;144:1087-94.

20. Skjaerpe T, Hatle L. Noninvasive estimation of systolic pressure in the right ventricle in patients with tricuspid regurgitation. Eur Heart J 1986;7:704-10.

21. Szwejkowski BR, Elder DH, Shearer F, et al. Pulmonary hypertension predicts all-cause mortality in patients with heart failure: a retrospective cohort study. Eur J Heart Fail 2012;14:162-7.

22. Malouf JF, Enriquez-Sarano M, Pellikka PA, et al. Severe pulmonary hypertension in patients with severe aortic valve stenosis: clinical profile and prognostic implications. J Am Coll Cardiol 2002;40:789-95.

23. Fayyaz AU, Edwards WD, Maleszewski JJ, et al. Global Pulmonary Vascular Remodeling in Pulmonary Hypertension Associated With Heart Failure and Preserved or Reduced Ejection Fraction. Circulation 2018;137:1796-810.

24. Weber C, Hung J, Hickling S, et al. Incidence, predictors and mortality risk of new heart failure in patients hospitalised with atrial fibrillation. Heart 2021;107:1320-6.

25. Genuardi MV, Shpilsky D, Handen A, et al. Increased Mortality in Patients With Preoperative and Persistent Postoperative Pulmonary Hypertension Undergoing Mitral Valve Surgery for Mitral Regurgitation: A Cohort Study. J Am Heart Assoc 2021;10:e018394.

26. Guazzi M, Dixon D, Labate V, et al. RV Contractile 
Function and its Coupling to Pulmonary Circulation in Heart Failure With Preserved Ejection Fraction: Stratification of Clinical Phenotypes and Outcomes. JACC Cardiovasc Imaging 2017;10:1211-21.

27. Kapłon-Cieślicka A, Lund LH. Atrial fibrillation in heart failure with preserved ejection fraction: a risk marker, risk factor or confounder? Heart 2020;106:1949.

28. Gersh BJ, Maron BJ, Bonow RO, et al. 2011 ACCF/ AHA Guideline for the Diagnosis and Treatment of Hypertrophic Cardiomyopathy: a report of the American College of Cardiology Foundation/American Heart Association Task Force on Practice Guidelines. Developed in collaboration with the American Association for Thoracic Surgery, American Society of Echocardiography, American Society of Nuclear Cardiology, Heart Failure Society of America, Heart Rhythm Society, Society for Cardiovascular Angiography and Interventions, and Society of Thoracic Surgeons. J Am Coll Cardiol 2011;58:e212-60.

29. Abramson SV, Burke JF, Kelly JJ Jr, et al. Pulmonary hypertension predicts mortality and morbidity in patients with dilated cardiomyopathy. Ann Intern Med 1992;116:888-95.

30. Authors/Task Force members; Elliott PM, Anastasakis A, et al. 2014 ESC Guidelines on diagnosis and management of hypertrophic cardiomyopathy: the Task Force for the Diagnosis and Management of Hypertrophic Cardiomyopathy of the European Society of Cardiology (ESC). Eur Heart J 2014;35:2733-79.

31. Magne J, Pibarot P, Sengupta PP, et al. Pulmonary hypertension in valvular disease: a comprehensive review on pathophysiology to therapy from the HAVEC Group. JACC Cardiovasc Imaging 2015;8:83-99.

32. Lam CS, Roger VL, Rodeheffer RJ, et al. Pulmonary hypertension in heart failure with preserved ejection fraction: a community-based study. J Am Coll Cardiol

Cite this article as: Zou T, Chen Q, Zhang L, Chen C, Ling Y, Liu G, Wang S, Pang Y, Xu Y, Cheng K, Zhou D, Zhu W, Ge J. Pulmonary artery pressure is associated with midterm major adverse cardiovascular events and postprocedure pericardial effusion in atrial fibrillation patients undergoing left atrial appendage occlusion. Ann Transl Med 2021;9(16):1324. doi: $10.21037 / \mathrm{atm}-21-3561$
2009;53:1119-26.

33. Murarka S, Lazkani M, Moualla S, et al. Left atrial anatomy and patient-related factors associated with adverse outcomes with the watchman device-a real world experience. J Interv Cardiol 2017;30:163-9.

34. Munir MB, Khan MZ, Darden D, et al. Pericardial effusion requiring intervention in patients undergoing percutaneous left atrial appendage occlusion: Prevalence, predictors, and associated in-hospital adverse events from 17,700 procedures in the United States. Heart Rhythm 2021. [Epub ahead of print]. doi: 10.1016/ j.hrthm.2021.05.017.

35. Sepahpour A, $\mathrm{Ng} \mathrm{MK}$, Storey P, et al. Death from pulmonary artery erosion complicating implantation of percutaneous left atrial appendage occlusion device. Heart Rhythm 2013;10:1810-1.

36. Zwirner J, Bayer R, Hädrich C, et al. Pulmonary artery perforation and coronary air embolism-two fatal outcomes in percutaneous left atrial appendage occlusion. Int J Legal Med 2017;131:191-7.

37. Hanazawa K, Brunelli M, Saenger J, et al. Close proximity between pulmonary artery and left atrial appendage leading to perforation of the artery, tamponade and death after appendage closure using cardiac plug device. Int J Cardiol 2014;175:e35-6.

38. Halkin A, Cohen C, Rosso R, et al. Left atrial appendage and pulmonary artery anatomic relationship by cardiacgated computed tomography: Implications for late pulmonary artery perforation by left atrial appendage closure devices. Heart Rhythm 2016;13:2064-9.

39. Lu C, Zeng J, Meng Q, et al. Pulmonary artery perforation caused by a left atrial appendage closure device. Catheter Cardiovasc Interv 2020;96:E744-E746.

(English Language Editor: A. Kassem) 